\title{
Germanica
}

\section{Philosophie de la musique dans le Docteur Faustus de Thomas Mann}

Musikphilosophie in Thomas Manns Doktor Faustus

\section{Christian Merlin}

\section{OpenEdition}

\section{Journals}

Édition électronique

URL : http://journals.openedition.org/germanica/2414

DOI : $10.4000 /$ germanica. 2414

ISSN : 2107-0784

\section{Éditeur}

Université de Lille

\section{Édition imprimée}

Date de publication : 30 juin 2000

Pagination : 115-129

ISBN : 9782913857032

ISSN : 0984-2632

\section{Référence électronique}

Christian Merlin, «Philosophie de la musique dans le Docteur Faustus de Thomas Mann », Germanica

[En ligne], 26 | 2000, mis en ligne le 07 mars 2014, consulté le 06 octobre 2020. URL : http:// journals.openedition.org/germanica/2414 ; DOI : https://doi.org/10.4000/germanica.2414

Ce document a été généré automatiquement le 6 octobre 2020.

(C) Tous droits réservés 


\title{
Philosophie de la musique dans le Docteur Faustus de Thomas Mann
}

\author{
Musikphilosophie in Thomas Manns Doktor Faustus
}

\author{
Christian Merlin
}

1 Ce n'est un secret pour personne, Thomas Mann a placé son dernier grand roman achevé sous le signe de la musique ${ }^{1}$. Il ne fait plus guère de doute non plus que, comme l'auteur s'en est expliqué dans le volume qu'il a consacré à l'élaboration de son ouvrage $^{2}$, il ne s'agit pas seulement d'un Künstlerroman au sens traditionnel, mais d'une somme à la portée historique, idéologique et philosophique considérable. Le narrateur Serenus Zeitblom fait assez clairement le lien entre le parcours intellectuel, artistique et humain de son ami et l'évolution de l'Allemagne vers la barbarie nazie et la guerre, contemporaine de la rédaction: il existe bel et bien un parallèle entre la vie du compositeur imaginaire Adrian Leverkühn et la chute de la culture allemande dans la bestialité. Mais quel rôle la musique joue-t-elle exactement dans cet arrière-plan ? N'at-elle qu'une fonction décorative, et les longues pages d'analyse à la précision musicologique millimétrée d'œuvres souvent imaginaires ne sont-elles qu'un effet de réel ? Joue-t-elle un rôle allégorique, exprimant sur un plan symbolique des théories que le héros aurait pu exposer dans une langue philosophique qui n'aurait pas été forcément adaptée au style romanesque ? Adrian Leverkühn serait ainsi un philosophe déguisé en musicien, hypothèse dans laquelle on serait volontiers conforté par des rapprochements tentants: ce personnage de fiction ne rappelle-t-il pas fortement Nietzsche, né comme lui près de Weißenfels, mort comme lui à 55 ans, en proie comme lui à dix ans de démence, victime comme lui d'une expérience bouleversante dans un bordel de Leipzig, musicien comme lui ? Mais s'arrêter à ces éléments anecdotiques serait bien insuffisant. La musique n'est pas qu'un substitut à la philosophie dans le Docteur Faustus, de même qu'elle n'est pas qu'une composante narrative brillamment agencée : sur le triple plan politique, esthétique et métaphysique, la pensée musicale de Thomas Mann, sous l'influence croisée de Kierkegaard, d'Adorno, de Benjamin et de la Naturphilosophie, trouve dans l'art des sons les clés d'interprétation du monde qui manquaient à l'intellectuel pour résoudre l'énigme nazie. 
Quelques jours après la capitulation de l'Allemagne, alors que le Docteur Faustus en est à la moitié de sa rédaction, Thomas Mann tient à la Bibliothèque du Congrès de Washington son célèbre discours "L'Allemagne et les Allemands " ${ }^{3}$. L'auteur y parle certes abondamment du sujet annoncé, mais il évoque aussi à plusieurs reprises la matière de son nouveau roman, et s'explique notamment sur la raison pour laquelle il a fait de son héros faustien un compositeur. Thomas Mann va jusqu'à s'étonner que Goethe n'ait pas déjà pensé à présenter Faust comme un musicien : il l'est forcément car la musique est par excellence un domaine démoniaque. Comme l'a montré Martin Huber ${ }^{4}$, c'est au Ou bien ou bien de Kierkegaard que Mann emprunte directement cette idée selon laquelle la musique est un " art chrétien de signe négatif $»^{5}$. Mann reprend ici à son compte une perplexité fortement teintée de fascination pour un mode d'expression artistique qui relève à la fois de l'ordre le plus rigoureux et du chaos le plus contraire à la raison, de la magie des nombres et de la passion démesurée, de l'abstraction mathématique et du mysticisme. Et c'est par cette dernière alliance que la musique se révèle à Thomas Mann comme un art spécifiquement allemand : «Si Faust doit être représentatif de l'âme allemande, alors il faut qu'il soit musicien; car les Allemands ont au monde un rapport abstrait et mystique, c'est-à-dire musical » (p. 1131).

Dans la droite ligne de sa critique des corrélations qui existent selon lui indéniablement entre l'art wagnérien et l'univers national-socialiste ${ }^{6}$, Thomas Mann constate que la musicalité allemande a conduit à une dissociation complète de l'activité socio-politique et la sphère spéculative, et à la primauté de cette dernière. Négligeant le contenu politique de l'œuvre d'art, les Allemands ont cru bon de se réfugier dans une conception purement esthétique et apolitique de la culture, et Thomas Mann rend cet aveuglement coupable de la voie tragique qu'ils n'ont pas hésité à emprunter à partir de 1933. Il reconnaît certes que l'Allemagne a donné à l'Occident sa musique la plus profonde et la plus importante, mais aussi qu'elle a dû " payer son âme musicale très cher dans une autre sphère: la sphère politique, celle de la cohabitation entre les hommes" (Deutschland, p.1132). Ce n'est pas la première fois que le mélomane passionné qu'était Thomas Mann fait part d'une certaine méfiance à l'égard d'un esthétisme déresponsabilisé. En 1939 déjà, lors d'une allocution au Congrès mondial des écrivains à New York ${ }^{7}$, il constatait "l'échec de la culture allemande purement esthétique » et mettait cette incapacité à assumer ses obligations politiques en relation avec l'avènement du nazisme, reprochant au citoyen allemand d'avoir toujours refusé " de reconnaître la politique comme partie intégrante de sa responsabilité humaine ». En 1940, dans sa fameuse lettre (en anglais) à l'éditeur de la revue Common sense, le romancier va plus loin encore dans cette condamnation d'une supposée innocence de l'œuvre d'art : « Le national-socialisme, c'est : “Je n'ai rien à faire de l'élément social. Ce que je veux, c'est du conte populaire." Cette formulation, à n'en pas douter, est la plus douce, la plus intellectuelle. En réalité, le fait est que si le national-socialisme est aussi une immonde barbarie, c'est parce qu'au royaume de la politique, les contes de fées deviennent des mensonges $»^{8}$.

4 Il n'est donc guère surprenant de retrouver, dans le roman proprement dit, semblables parallèles intégrés à la stratégie narrative poursuivie par l'auteur. Dès le chapitre VIII, lorsque les conférences du professeur de musique Wendell Kretzschmar évoquent la figure de Johann Conrad Beissel, des jalons sont posés pour un parcours qui va révéler progressivement les potentialités les plus douteuses de la musique. Kretzschmar y 
présente Beissel comme le fondateur, dans la Pennsylvanie du XVIII ${ }^{e}$ siècle, de la secte des anabaptistes du septième jour, mais aussi comme l'inventeur d'un système musical simple et accessible au moins instruit des fidèles, contrairement aux chorals européens trop complexes. Chaque membre de la secte était ainsi capable, grâce à des tables d'accords et à des grilles de notes, d'harmoniser ses propres chants religieux sur des rythmes d'une souplesse ondoyante. Deux indices attirent déjà l'attention du lecteur. Beissel est non seulement auteur d'une méthode musicale, mais aussi d'un traité sur le Sabbat et d'écrits didactiques empreints d'un symbolisme ésotérique : nous sommes résolument dans le domaine de l'irrationalisme superstitieux. Quant à son système d'écriture musicale, il repose sur une théorie en apparence banale de hiérarchie entre les notes, mais dont la terminologie est tout sauf innocente sur le plan politique puisqu'il y est question de " notes maitres » et de « notes serviteurs ». La musique y est considérée dans son rapport intime avec l'élément primitif, primordial, originel, cyclopéen - ce que scelle une allusion attendue à l'Anneau du Nibelung de Wagner -, et c'est à l'écoute de Kretzschmar qu'Adrian Leverkühn prend conscience que la culture n'est après tout peut-être qu'un phénomène transitoire et non une référence pour l'action humaine. L'idée que l'on puisse considérer la musique hors de son contexte culturel est d'ores et déjà insupportable au narrateur, l'humaniste Serenus Zeitblom, qui rétorque à son ami compositeur : «Mais il n'est d'autre alternative à la culture que la barbarie ». Fort de tout ce qu'il avait appris au cours des événements qu'il nous rapporte sur le mode du retour en arrière, le narrateur avait anticipé dès le chapitre II sur ce motif récurrent en nous faisant part de sa conclusion: "En dépit de toute la rigueur logique et morale qu'elle affecte parfois, [la musique] me semble bien plus ressortir à un monde spirituel dont je ne garantirai pas l'irréprochabilité sous l'angle de la raison et de la dignité humaine " (p.12). Cette méfiance est issue d'une longue tradition, comme Thomas Mann a pu s'en apercevoir lorsque Lion Feuchtwanger lui offrit les écrits d'Agrippa von Nettesheim comportant « un chapitre sur la musique, ou plutôt contre elle, plein d'invectives morales » (Entstehung, p. 134).

5 À la lumière de ces jugements, on ne compte plus les signes avant-coureurs savamment disposés par le romancier. Dès les études de Leverkühn, les conversations du cercle Winfried sont une préparation à celles, plus radicales encore dans leurs tonalités prénazies, qui ont lieu chez les Rodde, lors des réunions mondaines de la Rambergstraße. Cette fois encore, la musique est au centre des débats philosophiques, lorsque l'esthète cynique Breisacher prend plaisir à contredire ses interlocuteurs en considérant les apports de Bach et Palestrina comme un triomphe de la barbarie et non de la culture : en remplaçant la monodie (subjective) par la polyphonie (objective), la mélodie par l'harmonie, en généralisant le contrepoint, on n'a fait qu'abâtardir la force primitive et tribale des ancêtres. Cette stigmatisation de la musique cultivée, au profit d'un retour au rite conjuratoire, ne peut que paraître hautement suspecte au narrateur qui en perçoit avec acuité l'élément non pas tant conservateur que révolutionnaire (chapitre XXVIII). Les mêmes motifs connaîtront un paroxysme lors des discussions chez l'expert en éditions d'art Sixtus Kridwiss (chapitre XXXIV), lieu de rencontre d'une bourgeoisie cultivée qui a tout pour former le terreau des théories hitlériennes. Serenus Zeitblom y est profondément choqué par le dénigrement des valeurs bourgeoises issues de la philosophie des lumières qui formaient son propre horizon culturel, et il ne peut s'empêcher de voir dans la critique radicale de la tradition à laquelle se livrent les membres du cercle Kridwiss et dans leur volonté de retour à un chaos archaïque pré-musical une première manifestation de l'esthétisme irresponsable 
et antihumaniste qui conduira à la catastrophe nazie : «Ici, nul ne peut me suivre s'il n'a comme moi constaté dans son âme le voisinage de l'esthétisme et de la barbarie, de l'esthétisme en tant qu'avant-coureur de la barbarie » (p. 373), écrit le narrateur en se remémorant ces événements de 1919 alors qu'il est lui-même en train de suivre jour après jour l'effondrement de l'Allemagne nazie en guerre. On imagine son désarroi lorsqu'il retrouve des traces de ces débats chez Kridwiss jusque dans la musique d'Adrian Leverkühn, dont l'Apocalipsis cum figuris est en parfaite adéquation avec ce climat esthético-politique. Le narrateur en souligne l'écriture quasi-bruitiste, passant par le hurlement, le roulement de tambour «magique, fanatique et négroïde », et généralisant l'usage du glissando. Ce procédé, qui consiste à glisser d'une note à l'autre sans détacher les sons, revient dans l'ouvrage de Leverkühn, en particulier aux trombones, réminiscence peut-être du Sacre du Printemps de Stravinsky où les glissandi de trombones évoquent toute la sauvagerie d'un monde primitif. Or, Zeitblom, musicien élevé dans le respect de l'égalité des proportions de la musique classique, voit dans le glissando bien plus qu'un manquement aux canons de la forme mélodique traditionnelle : un "démonisme anticulturel, voire antihumain». Etendu aux timbales, le procédé lui semble encore plus terrifiant (on pense ici à Bartok, qui fut le premier à en généraliser l'usage grâce au recours à la timbale mécanique), mais c'est l'application du glissando à la voix qui lui semble le plus pervers: dans l'oratorio de son ami, il constate en effet une tendance à permuter les valeurs et à appliquer une écriture vocale aux instruments et une écriture instrumentale à la voix. À ce paradoxe s'en ajoute un autre : l'Apocalipsis cum figuris dépeint le sublime à l'aide de la dissonance la plus violente et l'enfer au moyen des harmonies tonales les plus suaves. Le narrateur voudrait voir dans cet art de la composition où le très archaïque et l'ultra-nouveau se rejoignent l'expression fidèle de la "courbure du monde». Cependant, plus de trois cents pages après les avoir évoquées pour la première fois, c'est aux spéculations «barbares » de Beissel que lui fait penser malgré lui l'oratorio de Leverkühn, Beissel et ses "notes serviteurs", puisque Zeitblom entend dans la musique de son ami une technique où « il n'y aurait plus une note libre » (p. 486). Musique barbare ou musique de la barbarie? Par la voix de son porte-parole, Mann pourrait sembler ici dans une impasse à laquelle la lecture d'Adorno lui permettra de trouver une issue.

C'est en 1941 que Theodor W. Adorno émigra en Californie, soit un an après que Thomas Mann s'y était lui-même installé. Adorno était solidaire et admiratif envers Mann depuis que celui-ci avait pris sur le phénomène wagnérien des positions critiques lucides qui lui avaient valu l'ire des cercles conservateurs comme des autorités nazies, le contraignant en dernier ressort à l'exil. Adorno avait d'ailleurs cité le texte de Mann Souffrance et grandeur de Richard Wagner (1933) dans son propre Essai sur Wagner (1937/38). Tout commença lorsque le philosophe, informé que Thomas Mann travaillait à une œuvre dans laquelle la musique jouait un rôle important, apporta début juin 1943 au romancier un ouvrage de Julius Bahle puis, deux semaines plus tard, lui confia sa propre étude sur Schoenberg qui allait devenir la première partie de sa Philosophie de la nouvelle musique (1949). Or, Thomas Mann nous apprend lui-même dans Die Entstehung des Doktor Faustus (p. 28) que c'est le 23 mai 1943 qu'il se mit à la rédaction proprement dite de son roman. Comme l'auteur le raconte (p.39), il fréquenta dès lors abondamment Adorno, qui devint son conseiller musical. Le chapitre XXII dans sa quasi-totalité ou l'analyse de la sonate op. 111 de Beethoven par Wendell Kretzschmar sont calqués, parfois au mot près, sur les textes d'Adorno, et le philosophe a pris une part active à la description des œuvres musicales fictives attribuées à Adrian Leverkühn 
comme le concerto pour violon ou le Chant de douleur du Dr Faustus. Certaines clés sont même décelables, comme le surnom que Kretzschmar donne à l'ariette de la sonate de Beethoven : "Wiesengrund », en fait la première partie (paternelle) du nom de famille d'Adorno. Et comme le rappelle Hans-Jürgen Schaal', la description du diable, qui connaît une transformation physique lorsqu'il en vient à évoquer avec Adrian les questions musicales, est un véritable portrait d'Adorno. Mais au-delà de ce plan purement anecdotique, c'est bien la philosophie de la musique d'Adorno qui éclaira les conceptions de Thomas Mann, que ce soit du reste pour la reprendre à son compte ou l'infléchir ${ }^{10}$.

7 C'est ainsi la fréquentation d'Adorno qui confortera Mann dans l'idée de donner à Adrian Leverkühn certains traits d'Arnold Schoenberg, au risque de se brouiller avec ce dernier, dont on sait la vexation à voir sa création ainsi détournée et caricaturée. C'est que la Philosophie de la nouvelle musique d'Adorno est en grande partie, malgré le militantisme de son auteur pour la seconde École de Vienne (Schoenberg, Berg, Webern), une critique de Schoenberg. Dès la première phase compositionnelle du musicien, sa période expressionniste et atonale, le philosophe décèle un goût suspect pour la régressivité. Fidèle à son système de l'antithèse et du paradoxe, chaque fois qu'Adorno souligne que l'expressionnisme exprime la solitude de l'homme moderne, il insiste en même temps sur sa dimension objective et anti-individuelle. De même, lorsqu'il évoque l'apparente modernité des œuvres où Schoenberg rompt avec les liens de la hiérarchie tonale, c'est pour ajouter que cet avant-gardisme apparent n'est en fait qu'un primitivisme: "L'origine de l'atonalité comme affranchissement complet de la musique des conventions, a précisément en cela quelque chose de barbare. [...] Le progrès lui-même, dans son refus de la convention, a quelque chose d'enfantin, de régressif. Les premières compositions atonales de Schoenberg, en particulier ses pièces pour piano op. 11, effrayaient plus par leur primitivisme que par leur complexité $»^{11}$. Adrian Leverkühn, lui aussi, dans les années qui précèdent la Première Guerre Mondiale, compose une œuvre comportant des parties «qui dédaignent tout rapport tonal » (p. 262). Mais il se sauve du reproche de primitivisme en recourant à une ironie distanciée qui permet l'imbrication et l'interpénétration entre les éléments archaïques et modernes de l'œuvre. Cette même ironie antipathétique dont, par un retournement très adornien, le narrateur mesurera le risque de régression lorsqu'il assistera au spectacle de marionnettes tiré de la Gesta romanorum, l'œuvre suivante de Leverkühn : "[...] plus que la sentimentalité étaient requises l'ironie, la raillerie ; elles assainissaient l'air, frondaient le romantisme, s'insurgeaient contre le pathos et le prophétique, la griserie des sons livresques, et se liguaient avec l'objectif et l'élémentaire ; autrement dit, il fallait redécouvrir la musique en tant qu'organisation du temps. Début épineux ! Car alors on risquait de tomber dans un faux primitivisme et donc dans un nouveau romantisme » (p.322). Ce nouveau primitivisme, c'est par exemple celui des œuvres néo-classiques de Stravinsky, l'un des principaux ennemis d'Adorno dans la Philosophie de la nouvelle musique (et ce n'est pas un hasard si Thomas Mann attribue à l'une des œuvres de Leverkühn l'exacte nomenclature instrumentale de L'Histoire du soldat de Stravinsky et Ramuz, la plus caustique, sautillante et dépouillée de leurs musiques de tréteaux : un violon, une contrebasse, une clarinette, un basson, une trompette, un trombone et la percussion). Sous couvert de musique populaire, Adorno et avec lui Mann voyaient poindre le spectre du populisme. Or, pour Leverkühn, «l'art est esprit et l'esprit n'a pas à se sentir engagé envers la société, la collectivité. [...] Un art qui "va au peuple", qui fait sien les besoins de la foule, du petit bourgeois, du vulgaire, tombe à 
l'indigence " (p.323). Nous sommes dans l'impasse: l'art socialisé est indigent, l'esthétisme déconnecté du politique est barbare...

8 Confronté à ce cul-de-sac, Thomas Mann imagine la voie originale que son héros empruntera pour réaliser son propre idéal musical au risque de sombrer dans le diabolisme; Adrian Leverkühn expose au chapitre XXII à Serenus Zeitblom le système musical qu'il a inventé, et qui permet d'établir une parfaite rigueur sui generis : il s'agit du dodécaphonisme. Avec virtuosité, Thomas Mann intègre ici dans la fiction de son roman l'invention de Schoenberg avec laquelle la lecture d'Adorno l'a familiarisé (c'est devant les protestations insistantes du compositeur que Mann devra insérer en dernière page de son roman un avertissement rappelant que la forme musicale décrite au chapitre XXII est la propriété intellectuelle du musicien au demeurant mortifié que sa méthode de composition fût présentée comme l'œuvre d'un artiste diabolique). C'est dans les années vingt que Schoenberg commença à composer selon le système qu'il avait lui-même mis au point, la musique sérielle (système de composition reposant sur l'utilisation exclusive des séries dodécaphoniques: les douze degrés de la gamme chromatique y sont utilisés sous forme de séries au cours desquelles aucune note ne peut être répétée avant que les onze autres n'aient été énoncées). Or, dans la Philosophie de la nouvelle musique, Adorno n'épargne pas non plus cette période de la création schoenbergienne. Pour lui, en inventant et imposant des règles formelles aussi abstraites et rigides, Schoenberg, loin de se libérer de la subjectivité, s'est rendu esclave d'une forme d'apparence objective mais d'origine parfaitement subjective. Et c'est pour lui la définition même de la supersitition: "un système clos et en même temps impénétrable à lui-même ». Cette dialectique entre servitude et liberté du système se reflète à l'identique dans la discussion entre Leverkühn, convaincu qu'il est « lié par la contrainte d'un ordre qu'il s'est imposé lui-même, donc libre», et Zeitblom, qui considère l'abolition totale du lien entre consonance et dissonance visé par son ami comme une utopie et une simple spéculation mathématique non dénuée de superstition, comme l'atteste la référence constante d'Adrian au carré magique.

9 Lorsque Thomas Mann découvrit la pensée d'Adorno, il était à la recherche de la " musique déchaînée " promise au monde par Adrian Leverkühn. Jusqu'alors convaincu que cette musique sans liens revêtirait des sonorités dionysiaques, il changea d'avis après avoir lu les pages d'Adorno sur la phase post-dodécaphonique de Schoenberg et sur les dernières œuvres de Beethoven. Ce sont désormais des catégories comme la souveraineté du langage et l'affranchissement du matériau qui dominent. Pour Adorno en effet, Schoenberg comme le dernier Beethoven (celui, par exemple, de la sonate pour piano op. 111 ou des ultimes quatuors à cordes), sont parvenus à une maîtrise nouvelle sur le matériau en le fragmentant, en le rompant pour mieux le renforcer, « et se sont soumis à lui tout en l'adaptant à leurs intentions au point d'interdire toute distinction entre subjectivité et objectivité » (Philosophie de la nouvelle musique, p. 113). Désormais, la question centrale pour Mann comme pour Adorno (re)devient la place du sujet et de l'expression dans l'organisation abstraite qu'est la musique : « comment la construction peut-elle devenir expression sans s'abandonner douloureusement à la subjectivité plaintive ?", demande Adorno (p. 96) en évoquant les dernières œuvres de Schoenberg. Apparemment, c'est précisément ce que le narrateur du Docteur Faustus concède à l'œuvre ultime d'Adrian Leverkühn: "Le créateur du Chant de douleur du DrFaustus peut, dans le matériau organisé par avance, s'abandonner sans entraves, insoucieux de la structure déjà prévue, préétablie, s'abandonner à la subjectivité, et de la sorte, son œuvre la plus rigoureuse, une œuvre où le calcul est poussé à l'extrême, se trouve être 
en même temps purement expressive»(p.487). Or, ce chant de la plainte, ultime paradoxe, est confié aux seuls instruments, ce qui fonde Zeitblom à parler de «la contre-partie de l'hymne à la joie, la négation fondamentale de ce passage de la forme symphonique à l'exaltation vocale» (p. 489). Cette idée d'une révocation nécessaire de la Neuvième Symphonie de Beethoven et de son message des lumières tient à l'évidence beaucoup d'Adorno, qui définissait lui-même sa Philosophie de la nouvelle musique comme une "digression à la Dialectique de l'Aufklärung» (avant-propos, p. 11). Mais elle doit aussi beaucoup à Walter Benjamin, dont Adorno avait offert à Thomas Mann L'Origine de la tragédie allemande $e^{12}$, ouvrage où l'auteur explique précisément (p.254) que le deuil conduit au mutisme. Adorno considérait la musique nouvelle comme une victime sacrificielle qui a renoncé au sens pour faire contre-poids à un monde qui a lui-même perdu tout sens : «l'inhumanité de l'art doit dépasser celle du monde pour la cause de l'humain» (p. 125). C'est le point nodal où ce qui était jusqu'ici un débat politique éthique et esthétique prend une dimension religieuse.

Adorno proposait en quelque sorte de combattre le mal par le mal, autrement dit d'opposer à une négativité une autre négativité. Sa vision restait donc foncièrement critique, voire nihiliste. Thomas Mann établit un diagnostic comparable mais en tire des conclusions différentes car il n'abandonne jamais totalement l'espoir d'une rédemption. Claude Gandelman écrit "qu'Adrian Leverkühn meurt d'un excès d'abstraction $»^{13}$. C'est ne prendre en compte qu'un aspect partiel de son parcours artistique. Certes, l'enfance du héros et son apprentissage sont placés sous le signe des jeux géométriques de son père avec les cristaux de glace. Au chapitre III, le père Leverkühn est fasciné par les éléments cristallins dont les composantes chimiques s'organisent en complexes lumineux plus beaux et inattendus les uns que les autres. Le caractère spéculatif et artificiel de ces expériences familiarise le jeune enfant avec la conviction que l'on peut manipuler des matières "absolument non-organiques". Parallèlement, Adrian est ainsi mis en contact avec le froid de la glace. On retrouvera ces influences dans les conceptions musicales qu'il expose à Serenus Zeitblom lors de leurs nombreux échanges : le fait que la musique est une création abstraite et que la forme musicale n'est pas organique, le fait aussi que le froid est principe créateur. Ainsi, au retour de la conférence de Kretzschmar, lorsqu'Adrian fait l'éloge des instruments «inorganiques» au profit de la voix humaine, trop chaleureuse, et du besoin qu'il y a à contrebalancer par une organisation sévère la tendance naturelle de la musique à s'adresser à l'instinct plus qu'à l'intellect : « La règle, toute règle produit un effet réfrigérant et la musique a en elle tant de chaleur propre, de chaleur d'étable, de chaleur de vache dirais-je, qu'elle peut avoir besoin de toutes sortes de règles réfrigérantes et elle-même du reste y a toujours aspiré » (p.71). Un peu plus loin, Leverkühn parle de "réfrigération ascétique", de même que, dans sa lettre à son professeur Kretzschmar au chapitre XV, il écrivait: «Je crains, cher maître et ami, d'être un vilain type car je n'ai en moi aucune chaleur» (p. 131).

11 Contaminé par le nihilisme, Adrian Leverkühn est-il donc inaccessible à toute idée de salut, incapable de chaleur et d'amour? Méphistophélès lui-même conclut son long dialogue du chapitre XXV en mettant clairement le froid qui entoure Leverkühn sur le compte d'une proximité avec le diabolique: "L'amour t'est interdit parce qu'il réchauffe. Ta vie devra être frigide» (p.250). Placée ainsi sous le signe du tiraillement entre humanité et inhumanité, la musique du Docteur Faustus devient le lieu d'un combat philosophique hérité de l'humanisme de la Renaissance et de la Naturphilosophie, avec la rédemption pour enjeu. Il est vrai qu'outre Adorno et 
Benjamin, une source de documentation essentielle fut pour Thomas Mann le Dürer und seine Zeit de Wilhelm Waetzoldt (1936), comme Dieter Borchmeyer le rappelle dans une des études les plus pénétrantes jamais consacrées au Docteur Faustus ${ }^{14}$. Borchmeyer nous invite à considérer comme une clé d'interprétation pour le roman de Mann la gravure d'Albrecht Dürer Melencolia I, où figurent entre autres, le compas, la balance, le polyèdre, le sablier et... le carré magique qui est précisément accroché dans le cabinet de travail Leverkühn, au-dessus du piano. Dürer considérait la géométrie comme la science par excellence et se définissait lui-même comme un mélancolique, jugement confirmé par Melanchton qui parlait de sa melancholia generosissima, et bien plus tard par Walter Benjamin qui, toujours dans l'Origine de la tragédie, établit une parenté entre la science arithmétique et la mélancolie, au sens des quatre tempéraments de l'Antiquité grecque (sanguin, flegmatique, colérique et mélancolique). S'appuyant sur la précision scientifique des définitions données par l'ouvrage Saturn und Melancholie. Studien zur Geschichte der Naturphilosophie und Medizin, der Religion und der Kunst de Raymond Klibansky, Erwin Panofsky et Fritz Saxl (Francfort 1990), Borchmeyer n'a guère de peine à identifier en Adrian Leverkühn la figure du créateur mélancolique : il en a les humeurs biliaires (déjà attribuées par Thomas Mann à Wallenstein dans son Versuch über Schiller), il est proche de la terre par son père, comme en témoignera son retour à un cadre de vie identique à celui de son enfance, il a pour compagnon un chien (comme le personnage de Dürer), qui porte du reste un nom diabolique (Kaschperl), il a froid, il souffre de migraines (comme Nietzsche, lui-même auteur d'un An die Melancholie écrit en 1871, soit un an après s'être procuré une reproduction de Melencolia I de Dürer qu'il avait offerte à Cosima Wagner). Et lorsque, au chapitre XXVIII, on apprend qu'Adrian met en musique des poèmes anglais, on n'est guère surpris que l'un d'entre eux soit l'ode À la mélancolie de Keats.

Ce qui est nouveau, c'est l'articulation opérée par Thomas Mann entre la conception mélancolique du monde et la musique. Depuis que la Bible attribua à la harpe de David la capacité de chasser les mauvais esprits qui hantent Saül, la musique était traditionnellement associée au versant positif, à la chaleur du tempérament sanguin. Mann la situe cette fois du côté de la magie noire, et ce n'est pas un hasard si Adrian Leverkühn compare directement la composition aux sciences occultes, reprenant à la lettre le vocabulaire de l'alchimie: "J'ennoblirai la prima materia en lui apportant l'adjonction du magisterium et en l'épurant, par l'esprit et par le feu, dans force cornues et alambics. Tâche splendide" (p.133). Cette "tâche splendide" qui a pour don d'exalter le frigide compositeur, c'est celle qui consiste à faire se rejoindre raison et magie, comme l'expliquera Leverkühn au fameux chapitre XXII, répondant aux objections de Zeitblom qui voit dans les constellations de notes du sérialisme plus d'astrologie que de géométrie ${ }^{15}$. Au chapitre XIII, décrivant l'étrange chargé de cours en théologie Eberward Schleppfuß, Serenus Zeitblom avait tiré la conclusion selon laquelle la «théologie incline par nature à la démonologie» (p. 100). On découvre maintenant que la musique connaît un processus exactement similaire qui mène de la clarté à l'obscurité, comme Thomas Mann le concède dans Die Entstehung des Doktor Faustus : « La clarification constructive, mue par une nécessité objective, qui caractérise la musique, régresse pour des raisons tout aussi objectives vers l'obscurité de la mythologie». L'importance du motif de la mélancolie prend alors tout son sens: Walter Benjamin nous apprenait en effet (p. 162) que la mélancolie, bien que mue par une aspiration au contact avec Dieu, était volontiers soupçonnée d'être en contact avec «l'esprit des enfers ». Le combat est alors spécifiquement religieux, entre la musica angelica et la 
musica diabolica, et si Serenus Zeitblom, avec sa viole d'amour, parlait de la musique comme d'un « don de Dieu » ou d'un « art divin », c'est bien d'un don du diable ou d'un art diabolique qu'il faudrait parler si l'on en croit la description pathétique qu'un Leverkühn atteint par la démence fait à l'assemblée médusée de son pacte avec le démon, au chapitre 47 : au contraire de Palestrina, inspiré par le concert des anges, ce sont des voix démoniaques qui semblent dicter à Adrian son œuvre ultime.

De cela aussi, Zeitblom a eu assez tôt l'intuition, en particulier dans les pages où il évoquait l'ironie consubstantielle au style de son ami. On se souvient par exemple de la contrariété du narrateur lorsque, pour sa fantaisie pour orchestre en un mouvement composée entre fin 1913 et début 1914, Leverkühn préféra le titre Merveilles du Grand Tout à Symphonia Cosmologica que préconisait Zeitblom : comme toujours, le compositeur a privilégié la raillerie, la parodie, clairement identifiée par Serenus comme une "causticité satanique» (p. 275) qui s'en prend non seulement à l'univers mais au médium de celui-ci : la musique. Nouveau trait du mélancolique : il rit certes, mais son rire n'est pas de l'humour, il est sardonique, Serenus Zeitblom y voit une ivresse diabolique, une "dissolution orgiaque de la rigueur vitale ». C'est à Kierkegaard que nous ramènent ces pages, et notamment à sa thèse sur Le Concept d'ironie (1841), dont Thomas Mann avait eu connaissance grâce à l'ouvrage d'Adorno Kierkegaards Konstruktion des Ästhetischen (Tübingen, 1933), où le philosophe scandinave est d'ailleurs expressément présenté comme un mélancolique (p.138/139) : on pense en particulier à la distinction qu'opère Kierkegaard entre l'humour, qui est expression indirecte de la vérité et donc empreint de positivité, et l'ironie, renversement négatif des normes humaines. Quant au fait que la conclusion du Chant de douleur du Docteur Faustus soit purement orchestrale et non plus vocale, peut-être faut-il y voir une réminiscence de la conception médiévale opposant la musica instrumentalis, qui est œuvre du diable, et la musica mundana, qui est coelestis et divina. Cette inversion, le roman de Thomas Mann nous y prépare dès ses toutes premières pages : alors que Leverkühn montre à son ami comment l'on peut rapprocher des tonalités en apparence très éloignées par le procédé de l'enharmonie (notes synonymes changeant non de hauteur mais de fonction dans les relations harmoniques), il prononce la phrase clé : "Sais-tu ce que je trouve? La musique est l'ambiguïté érigée en système ».

Or, c'est précisément ce double sens de la musique - qui dépasse de beaucoup la traditionnelle opposition entre Dionysos et Apollon -, qui peut fournir la lueur d'espoir dont Thomas Mann avait besoin pour laisser entrevoir une rédemption possible d'Adrian Leverkühn, et donc de notre époque. Si la musique est traversée par des influences bénéfiques (ordre, raison, foi, progrès, harmonie céleste, équilibre entre la forme et le sens) autant que malfaisantes (chaos, irrationnel, superstition, régression, cacophonie infernale, basculement de l'abstraction des nombres dans la magie), rien ne dit que la victoire définitive du mal soit une fatalité. Leverkühn lui-même ne se montre pas constamment résigné à vivre le cœur glacé par sa proximité avec le démon. Certes, ses premières tentatives de redonner une certaine place au sentiment sont bien maladroites: nous avons suffisamment vu ce que l'innocence et l'humanité qu'il préconise au chapitre XXXI avaient de trompeur et de démagogique, et même ses amis proches, au lieu de s'en réjouir, s'inquiétaient de cette tendance nouvelle à la sentimentalité qui «ne s'accordait pas à lui » (p. 323). «Je n'aimerais pas entendre de toi une œuvre d'inspiration humaine ", va jusqu'à lui dire Rudi Schwerdtfeger. C'est une tout autre voie qu'empruntera Adrian Leverkühn dans le Chant de douleur du Docteur Faustus, qui n'est ni celle du primitivisme barbare, ni celle de l'ironie sardonique, ni 
celle de la sentimentalité complaisante. C'est tout simplement celle du silence. Thomas Mann voulait y voir une source d'espérance, au risque de paraître trop optimiste aux yeux d'Adorno: dans Die Entstehung des Doktor Faustus, il raconte comment son "véritable conseiller privé", comme il aimait à l'appeler, lui avait conseillé de reprendre son chapitre XLVI, exagéré dans son espérance naïve d'un réconfort. Mais malgré les modifications consenties par Thomas Mann, le romancier a persisté à contrecarrer la négativité radicale de la pensée d'Adorno, un peu comme Wagner n'a jamais complètement abdiqué sa croyance en un progrès, même au plus fort de l'influence de Schopenhauer. Carl Dahlhaus n'a donc pas tort de faire une forte distinction entre l'Adorno conseiller musical de Thomas Mann et l'Adorno auteur de Philosophie de la nouvelle musique ${ }^{16}$. Loin d'être une négation de son message de fraternité, la révocation de la Neuvième Symphonie de Beethoven à laquelle se livre Leverkühn a en fait valeur de témoignage, d'avertissement: de même que Thomas Mann ne concevait pas que l'on pût entendre la musique universaliste de Fidelio dans l'Allemagne de Himmler sans avoir envie de quitter la salle en se prenant la tête entre les mains, il considérait une reprise de la Neuvième Symphonie comme déplacée dans un contexte de barbarie. On rejoint une dernière fois l'enseignement de Kierkegaard, pour qui il existe une vertu positive du désespoir ${ }^{17}$. Et de même que Kierkegaard considérait en dernier ressort le silence comme valeur suprême d'authenticité ${ }^{18}$, tout ce que peut faire Adrian Leverkühn pour soustraire sa musique à l'influence du démon, c'est la faire taire, «mais le son encore en suspens dans le silence, le son qui a cessé d'exister, que l'âme seule perçoit et prolonge encore et qui tout à l'heure exprimait le deuil, n'est plus le même. Il a changé de sens, et à présent il luit comme une clarté dans la nuit ».

\section{NOTES}

1. Nous citerons à partir de l'édition Fischer Bücherei (Francfort 1967). Les citations tirées du Docteur Faustus seront données dans la traduction française de Louise Servicen (Paris, Albin Michel, 1950). Toutes les autres citations seront traduites par nos soins.

2. . Thomas Mann: Die Entstehung des Doktor Faustus, paru en 1949, cité dans l'édition de 1966, Francfort (S. Fischer Verlag).

3. . Thomas Mann: "Deutschland und die Deutschen", vol. XI des Gesammelte Werke in dreizehn Bänden, Francfort 1974.

4. . Martin Huber : "Musikalität und Nationalsozialismus in Thomas Manns Doktor Faustus », in : ders.: Text und Musik. Musikalische Zeichen im narrativen und ideologischen Funktionszusammenhang ausgewählter Erzähltexte des 20. Jahrhunderts. Francfort-BerlinBerne-New York-Paris-Vienne (Peter Lang, Münchener Studien zur literarischen Kultur in Deutschland, Band 12) 1992, p. 107.

5. . Sören Kierkegaard: Entweder-Oder, en particulier les pages sur l'érotique musicale, Munich 1975, p. 89.

6. . Christian Merlin: "Thomas Mann et Richard Wagner", in: Radio Classique, mensuel, juillet 1997.

7. . Thomas Mann : An die gesittete Welt. Politische Schriften und Reden im Exil. Francfort 1986, p. 916. 
8. . Thomas Mann : "To the editor of Common Sense ", in : ders. : Wagner und unsere Zeit. Aufsätze, Betrachtungen, Briefe, hrsg. von Erika Mann. Francfort (Fischer Taschenbuch Verlag) 1990, p. 158.

9. . Hans-Jürgen Schaal : « Thomas Manns Musikerroman Doktor Faustus. Der Einfluß von Arnold Schönberg und Theodor W. Adorno », in : «Das Orchester ", n¹-1998, p. 2 sq.

10. . Thomas Mann rapporte même, dans Die Entstehung des Doktor Faustus (p. 109), qu'il a écrit une lettre d'une dizaine de pages pour s'excuser tant bien que mal auprès d'Adorno pour tous ses emprunts.

11. . Theodor W. Adorno : Philosophie der neuen Musik, Francfort (Suhrkamp Taschenbuch) 1976, p. 46.

12. . Walter Benjamin: Ursprung des deutschen Trauerspiels, Francfort 1963. Thomas Mann mentionne l'ouvrage à deux reprises dans Die Entstehung des Doktor Faustus, p. 36 et 134.

13. . Claude Gandelman: "Abstraction et empathie. Présence d'un thème esthétique dans l'œuvre de Thomas Mann », in : Études Germaniques, avril-juin 1975, p. 179.

14. . Dieter Borchmeyer : "Musik im Zeichen Saturns. Melancholie und Heiterkeit in Thomas Manns Doktor Faustus », in : Thomas Mann Jahrbuch, Band 7, 1994, p. 123.

15. Encore un héritage d'Adorno, qui écrit: «Le jeu de nombres de la technique dodécaphonique et la contrainte qu'il exerce est apparenté à l'astrologie » (Philosophie der neuen Musik, p. 67).

16. . Carl Dahlhaus, « Fiktive Zwölftonmusik », in : Musica 37, 1983, p. 245.

17. . Cf. Jean Wahl, Études kierkegaardiennes, Paris, Vrin, 1974, p. 71.

18. . Ibid., p. 273.

\section{RÉSUMÉS}

Si Adrian Leverkühn, le héros faustien du Docteur Faustus de Thomas Mann, est un musicien, c'est parce que, pour l'auteur, il ne pouvait en être autrement : Faust ne peut être que musicien car la musique est, par excellence, un domaine démoniaque, un "art chrétien de signe négatif », par référence à Kierkegaard. La méfiance du narrateur envers un art qui mêle «l'ordre le plus rigoureux et le chaos le plus contraire à la raison » est transposé sur le plan politique : la musique allemande est rendue coupable de l'esthétisme purement spéculatif et apolitique, déconnecté de la réalité sociale, qui a préparé la voie au nazisme. Thomas Mann tente de faire ici la synthèse de la Philosophie de la nouvelle musique d'Adorno, qui était à l'époque de la rédaction son conseiller musical. Il stigmatise notamment tout ce qui participe du retour à un primitivisme irrationnel qui conduit à la barbarie en renonçant à l'humanisme des lumières (ce n'est pas un hasard si l'ultime œuvre de Leverkühn est présentée comme la « révocation de la Neuvième Symphonie de Beethoven »). Sont visés ici, paradoxalement, tant Stravinsky et ses archaïsmes, que Schoenberg et le dodécaphonisme, construction intellectuelle en apparence objective, mais qui relève en fait d'un mysticisme des chiffres guère éloigné de l'astrologie et de la superstition. C'est ici que le roman de Mann quitte la sphère éthique et esthétique pour entrer dans la sphère religieuse : Adrian Leverkühn, qui possède tous les traits (y compris diaboliques) du « mélancolique » tel que Walter Benjamin les a théorisés, cherche aussi la voie du salut et, surmontant la négativité radicale d'Adorno, se rapprochera de Kierkegaard en affirmant dans sa musique la positivité du désespoir et du silence. 
Daß Adrian Leverkühn, der faustische Held von Thomas Manns Doktor Faustus, ein Komponist ist, kommt nicht von ungefähr; laut Thomas Mann könne Faust nur Musiker sein, weil die Musik nach Kierkegaard dämonisches Gebiet schlechthin sei, «christliche Kunst mit negativem Vorzeichen ». Daß der Erzähler einer Kunstform gegenüber höchst mißtrauisch ist, die zugleich «berechnetste Ordnung und chaosträchtige Widervernunft » ist, findet im politischen Bereich seinen Niederschlag: Die deutsche Musik wird des rein spekulativen, apolitischen, jeder sozialen Realität fremden Ästhetizismus bezichtigt, der für den Wegbereiter des Nationalsozialismus gehalten wird.Hier versucht Thomas Mann, die Philosophie der neuen Musik seines damaligen musikalischen Beraters Adorno zu synthetisieren. So brandmarkt er alles, was zum irrationalen Primitivismus beiträgt, der zur Barbarei und zum Verzicht auf den Humanismus der Auklärung führt (Leverkühns letztes Werk wird nicht zufällig als «Widerruf » der Neunten Symphonie Beethovens bezeichnet). Paradoxerweise werden hier sowohl Stravinskys Archaismen als auch Schoenbergs Zwölftonmusik gemeint : letztere erscheine zwar als objektive Konstruktion, gehöre aber in der Tat zur Zahlenmystik und sei irgendwie mit Astrologie und Aberglauben verwandt. Hier geht Manns Roman deutlich von der ethisch-ästhetischen Sphäre zur religiösen über: Adrian Leverkühn, der alle Züge des von Walter Benjamin theorisierten « Melancholikers » trägt, d.h. auch teuflische Züge, ist auch auf der Suche nach dem Weg zur Erlösung. Er wird Adornos radikale Negativität überwinden, indem er in Anlehnung an Kierkegaard die Positivität der Verzweiflung und vor allem des Schweigens bejaht.

\section{AUTEUR}

\section{CHRISTIAN MERLIN}

Université Charles-de-Gaulle - Lille III 